\title{
Rheological Model of Force Transmission through the Helmet and Concussion Sensitivity
}

\author{
Isiah Kendall, Anthony Vicini* and Tarun Goswami
}

Biomedical, Industrial and Human Factors Engineering, Wright State University, Dayton, OH, USA

\begin{abstract}
In contact sports, head-to-head collisions can lead to concussions, which pose serious health risks to players. This research aims to understand the force transfer from the helmet to the brain that causes concussions in collisions using a rheological model. Experimental data was gathered from players in the National Football League and testing of one type of helmet and padding. The rheological model was verified with published data and good correlation was achieved. Further sensitivity analysis of concussion risk was performed with respect to force, body weight, mass, and impact duration fit to normal and Weibull distributions using Monte Carlo simulations of impacts. A 50\% threshold for moderate concussion was found based on these physiological variables. Average weight and velocity values for an NFL player in a collision gave a $50 \%$ concussion risk to a helmet to helmet impact that has a deceleration over 6.365 ms or less. Analysis of children ranging from 10 to 15 years of age was also conducted with the assumption of identical equipment to NFL players due a dearth of other research into the properties of equipment used by children. As the equipment is assumed to decrease in quality over time, this established an upper bound to the tolerance values for children. For a 50th percentile weight 10 year old male or female child, this gives thresholds of 2.483 or $2.573 \mathrm{~ms}$ respectively.
\end{abstract}

Keywords: Football; Rheological model; Concussion; Injury risk; Sensitivity analysis; Head impact

\section{Introduction}

The number of athletes participating in collision sports is constantly growing. It is estimated that 1.28 concussions result from every 1000 contact exposure in collegiate and NFL games [1]. These account for $50 \%$ of all concussion statistics, with a further $25 \%$ of all concussions attributable to other sports [2]. This translates to 1.6-3.8 million sports related concussions per year in the United States [3]. When football player do experience an injury suspected of causing a concussion, current protocols allow them to return to the field if they have no loss in memory or cognition despite the more than doubled risk of concussion chance in short term, repeated impacts [4]. Additionally, there is no commonly accepted method to conduct concussion evaluation when dealing with players who have had repeated concussions [3].

It is well known that concussions, especially when repeated, can cause changes in thinking and mental aptitude. In more serious impacts, concussions and other forms of traumatic brain injury (TBI) are a leading cause of death from sporting related injuries [5]. It is also believed that both genders are equally prone to concussions, with males experiencing most concussions in football and females experiencing most concussions in soccer than other sports [2]. During an impact, force is evoked which causes a rotational force to propagate through the head and into the neck. Studies show average football impacts commonly range between 5654 and $7608 \mathrm{~N}$ [6]. Impacts exceeding a $\mathrm{g}$-force of 70 or higher indicate for moderate concussion [7]. Pressures on the brain in excess of $234 \mathrm{kPa}$ in compression or $186 \mathrm{kPa}$ in tension can cause hematoma, contusions, and oedema [8].

Since children under 14 or 15 years of age usually play with limited protection or used helmets, this age group would be expected to be more prone to concussion. Few studies have studied the injury biomechanics of youths based on their body weights, velocities they achieve, equipment properties, and how often they collide. This is partially due to the variation in the body weight, height, and ambulatory power present in children as they grow. Additionally, there is little literature regarding the duration over which an impact's force is dissipated, complicating models. This paper seeks to understand how initial conditions of velocity and weight transform into g-forces in an impact before transferring to the brain for adults and children.

\section{Methods}

Anatomical properties of both children and adults were used in the simulation of a football collision. Adults were assumed to collide with a player of average mass traveling at the same speed as the test subject. Published data from 300 players composed of running backs and wide receivers from the 2012 roster were selected from the 32 teams in the NFL and their weights and 40 yard dash velocities were recorded. Speeds and weights were fit to a normal distribution and a Monte Carlo simulation was performed to predict the results of 500 collisions. The weight and velocity measurements were subjected to a Shapiro-Wilk test for normality in which insufficient evidence was found to reject normality in both cases $(\mathrm{P}=0.6853)$. In the case of youth simulations, a 50th percentile, 10 year old male was used as the target, with both players in a collision assumed to be traveling at the average velocities of children for their age as simulated by Sterken [9]. Collisions were simulated for children ranging from 50th to 90th weight percentiles from the ages of 10 to 15 years old. For simulation purposes, it was also assumed that the helmets used by the children reduced forces identically to that of helmets used by the NFL due to lack of research into specific properties of said equipment.

The helmet used here was of a weight typical to football helmets (1.4 $\mathrm{kg}$ ) was and assumed to be irrotational to the head. The force at impact

*Corresponding author: Anthony Vicini, Biomedical, Industrial and Human Factors Engineering, Wright State University, 2070 Zink Rd Apt 3C, Fairborn Dayton, OH, USA, 45435, Tel: 636448 9404, Fax: 937775 5009, E-mail: Vicini.3@wright.edu

Received September 25, 2014; Accepted November 25, 2014; Published December 03, 2014

Citation: Kendall I, Vicini A, Goswami T (2014) Rheological Model of Force Transmission through the Helmet and Concussion Sensitivity. Int J Neurorehabilitation 1: 132. doi:10.4172/2376-0281.1000132

Copyright: @ 2014 Kendall I, et al. This is an open-access article distributed under the terms of the Creative Commons Attribution License, which permits unrestricted use, distribution, and reproduction in any medium, provided the original author and source are credited. 
was obtained through analyzing the momentum of helmet collisions using an Arrhenius model of energy absorption, seen in equation 1, with the assumption of a linear elastic case. Stiffness data was taken from a standard Riddell helmet which had a foam padding thickness of $0.66 \mathrm{~cm}$ and a total padding area of $24.2 \mathrm{~cm}^{2}$ [10]. F is the maximum force at the point of impact. The reduced force and g-force at impact

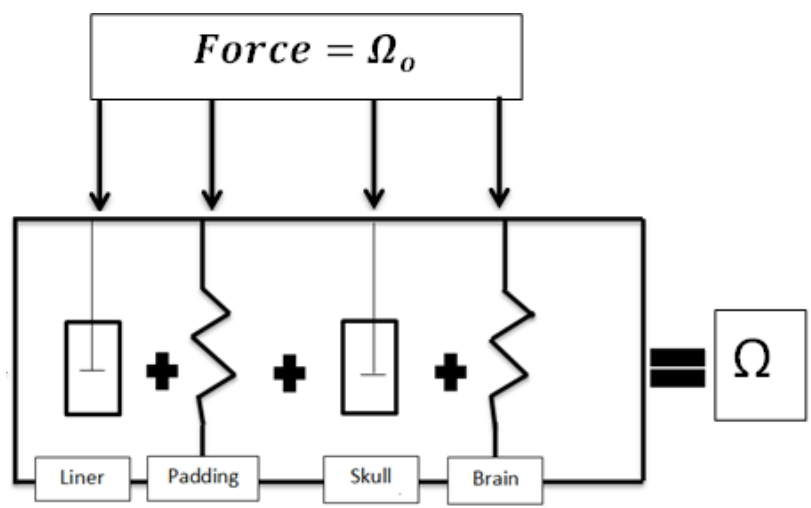

Figure 1: Dashpot and spring diagram of force absorption.

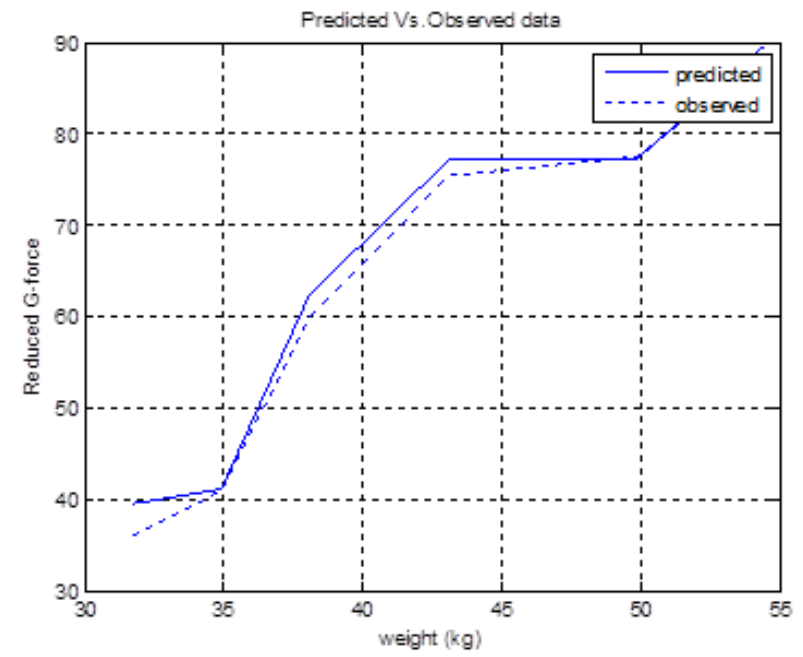

Figure 2: Rheological data vs. normal distribution for $2 \mathrm{~ms}$ collisions.

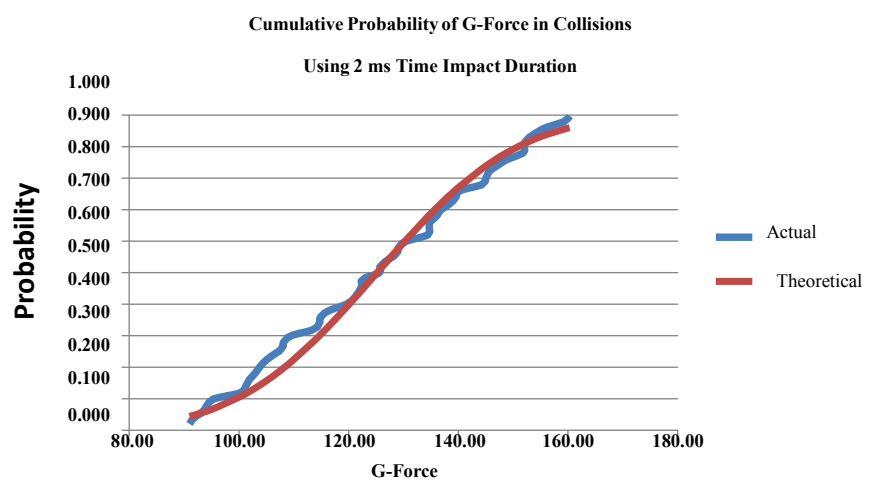

Figure 3: Injury probability based on Monte Carlo simulation with a $2 \mathrm{~ms}$ impact time. values are the forces experienced after transfer through the helmet. M1 and M2 represent the mass of the players colliding. $\mathrm{V}$ is the velocity of the player on whom the forces are being calculated [11]. $\mathrm{R}$ is the average radius of the players head. A is the area of impact, which was considered a constant value of $10 \mathrm{~cm}^{2}$ for this study. Tis time measured in milliseconds. The angular acceleration and angular velocity were calculated using the first cervical vertebra $(\mathrm{C} 1)$ as a pivot point as it provides the majority of the rotation to the skull [12]. By summing the different stiffnesses of the helmet shell, padding, and cranium, the total force transferred to the brain was determined by equation 2. A is the cross-sectional area of the material, $\mathrm{E}$ is the Young's Modulus of the material, $\mathrm{L}$ is the length of the material, and $\Delta \mathrm{L}$ is depth of the deformation. Once the force felt on the brain was found, the reduced $\mathrm{g}$-force was found by relating the incoming force and the reduced force.

$$
\begin{aligned}
& F=\left(1-\frac{M_{2}}{M_{1}+M_{2}}\right) \frac{1}{2} M_{2} V^{2} / R \times e^{-\frac{1}{2} A T} \\
& F_{\text {brain }}=\Delta L \sum \frac{A E}{L}
\end{aligned}
$$

Players were also analyzed using the Head Injury Criteria (HIC) for traumatic brain injury. The HIC equation was simplified for this by assuming a constant acceleration to get HIC $=\left(t^{2}-t^{1}\right) a^{2.5}$, where $t^{1}$ and $t^{2}$ are the start and end times respectively, and is the acceleration. After the HIC score was found for each impact, that data was converted into an injury chance using a normal distribution with a mean of 533 and a standard deviation of 91.94. This injury threshold delineates the expected values for a moderate brain injury, with anterograde amnesia lasting between 30 minutes and 24 hours and a Glasgow Coma Scale score between 9 and 12 [13]. Thresholds for more minor brain injury were not used as exact values for this are debated in literature, partly due to the fact that such injuries may not be apparent.

\section{Results}

\section{NFL and collegiate players}

The data generated by the governing equations of the Arrhenius model of energy absorption formed a novel rheological model of the system. The rheological model recreates the layers of the helmet in the form of a piston, dashpot, and spring mechanism. The resultant stiffness of the system was thus the summation of the stiffness of each component as seen in Figure 1. The sum of all the different material's stiffnesses was a value defined as $\Omega$.

Using these equations, a $15.45 \%$ decrease in the original force was found. The reduced force data predicted by the model was verified against the observed impact data by Moss and King in Figure 2. A paired-t test shows no significant evidence for difference between the data $(\mathrm{P}=0.947)$.

Using the Monte Carlo simulation, the likelihood of an impact reaching a certain force threshold was found after analysis of 1,000 generated data points based on the weights and velocities of NFL players. Normal and Weibull distribution models were used to display the frequency of each hit in terms of g-force given a two millisecond collision time in order to more easily see the effect of anatomical characteristics. The shape and scale parameters of the Weibull graph, $\alpha$ and $\beta$, were 7 and 134 respectively. The normal distribution had a mean and standard deviation of 125 and 20 respectively. It can be seen in Figure 3 that the g-force distribution from Moss and King was consistent with the normal distribution found here $(\mathrm{P}=0.6333)$. 

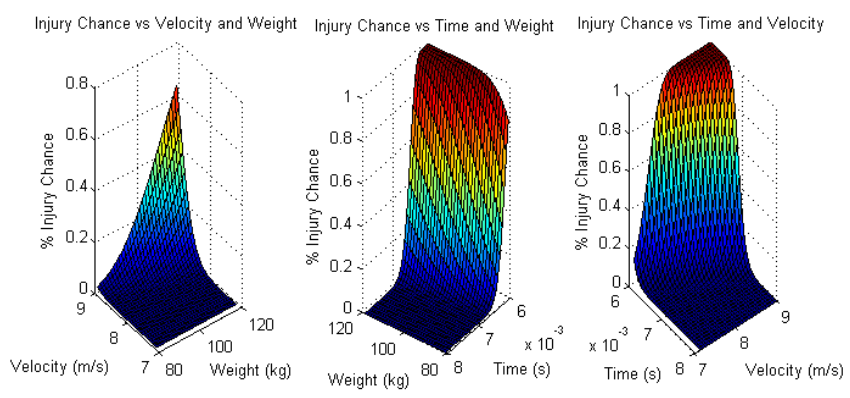

Figure 4: Change in injury chance given changes in velocity, weight, and time.

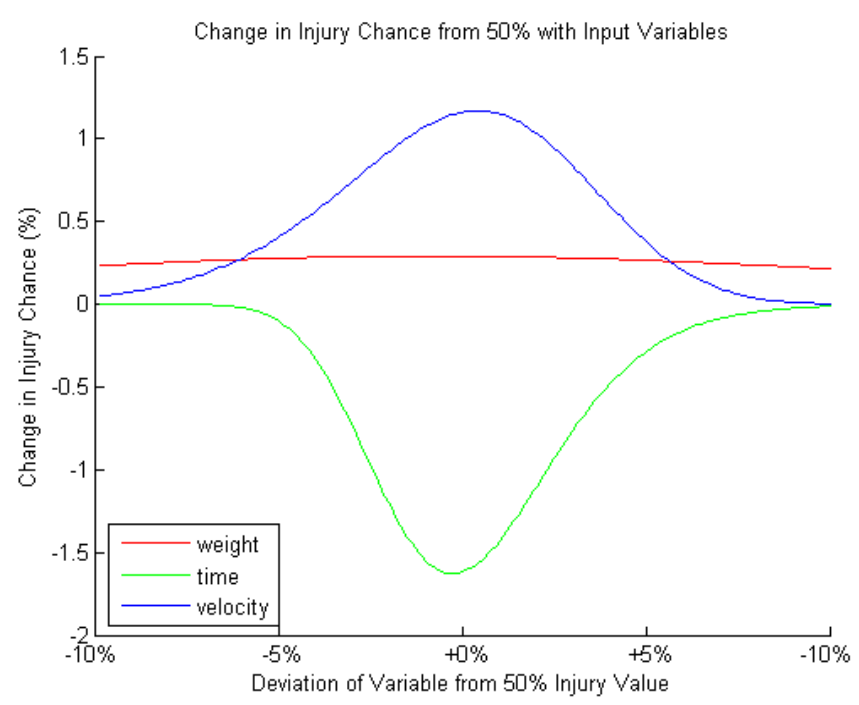

Figure 5: Time, velocity, and weight values corresponding to risk values.

\begin{tabular}{|c|c|c|c|}
\hline & & velocity & weight \\
\hline & time $\mathbf{( m s )}$ & $\mathbf{( m ~ s}^{-1} \mathbf{)}$ & $\mathbf{( k g )}$ \\
\hline $10 \%$ risk & 6.59 & 7.74 & 83.3 \\
\hline $50 \%$ risk & 6.37 & 8.07 & 101.1 \\
\hline $90 \%$ risk & 6.18 & 8.471 & 119.9 \\
\hline Sensitivity & 0.0040 & 0.007 & 0.360 \\
\hline
\end{tabular}

Table 1: Time thresholds for impact to evoke injury chance in male youths.

The HIC data generated from the players' masses and velocities listed in NFL charts were analyzed using the rheological force model to determine the real world correlation of the input terms on the prospective injury outcome. Initially, an effort was made with this data to choose a time value for the impact duration that resulted in data with a center of $50 \%$ injury rates to avoid saturation of data points. Weight, velocity, and impact duration were adjusted until a change in the injury rate of $1 \%$ was detected to yield sensitivity profiles for each statistic. The values needed to change the injury rate from the $50 \%$ mark for weight, velocity, and time were $0.360 \mathrm{~kg} / \%$ injury, $0.007(\mathrm{~m} / \mathrm{s}) / \%$ injury, and $-0.0040 \mathrm{~ms} / \%$ injury respectively. These values imply that a decrease in $0.36 \mathrm{~kg}$, a decrease of $0.007 \mathrm{~m} / \mathrm{s}$, or an increase in the impact duration of $0.0040 \mathrm{~ms}$ would change what would have been a collision with a $50 \%$ risk of concussion to a collision with a $49 \%$ risk of injury.

Figure 4 shows this process using the theoretical equations for injury based on impulse duration, weight, and velocity of NFL players, with one of these values held constant for each graph. Time was held constant at a level that showed the distribution of injury chance for weights and velocity ( $7 \mathrm{~ms})$. Weight and velocity were held at physiological values based on average NFL player's weights and velocities from obtained the NFL datasheets $(97.7 \mathrm{~kg}, 8.05 \mathrm{~m} / \mathrm{s})$. Figure 5 shows this effect with the variable in question isolated. The plots center each variable at the $50 \%$ threshold for injury, and the rate at which the derivative of the injury chance tends to zero corresponds with the sensitivity of the statistic in the calculations.

Based on these results, risk values for a moderate head injury due to a helmet to helmet hit were developed in Table 1 . The weight and velocity values were found by finding the impact duration that corresponds with the known injury rate $(0.128 \%)$ in the NFL and collegiate football and using that value for calculations [14]. The time risk value was created by finding the time that yields a $50 \%$ injury risk at average weights and velocities of NFL players. This value was found to be $6.56 \mathrm{~ms}$.

\section{Youth players}

The equations used for adult players were also extrapolated to predict minimum injury values with youths ranging in age from 10 to 15 years old, although it should be noted that actual injury thresholds may vary based on the efficacy of the equipment used. Injury calculations were made for collisions upon a 50th percentile weight, 10 year old of similar sex to the collider. The colliders were assumed to have the average velocities for their age and ranged from 50th to 90th percentile in terms of weight for ages 10 to 15 . The impulse duration required to evoke a certain probability of injury was found for collisions involving each unique weight percentile and age combination. Calculations showed threshold impact durations for a 50\% injury chance to be 3.38 $\mathrm{ms}$ for a 90th percentile weight, 15 year old male and $2.48 \mathrm{~ms}$ for a 10 year old, 50th percentile male. The same inputs for females give values of $3.45 \mathrm{~ms}$ and $3.00 \mathrm{~ms}$ respectively. Data points for a $10 \%$ risk level were graphed for males in Figure 6. It was found that for a given impact, female children are theoretically slightly more likely to receive a concussion than males based on average weights and velocities in all cases studied except 14 year old, 60th percentile, and 15 year old 5080th percentile weights.

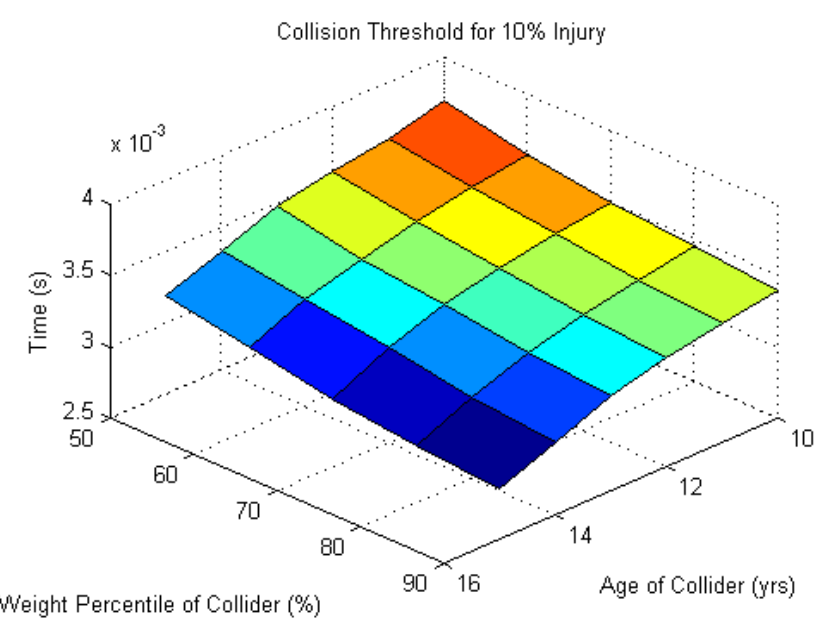

Figure 6: Time thresholds for impact to evoke $10 \%$ injury chance in male youths. 


\section{Discussion}

\section{NFL and collegiate players}

NFL data analysis using injury chance data derived from HIC scores revealed a $50 \%$ injury rate prediction for concussion in a direct helmet to helmet collision for velocities of $8.07 \mathrm{~m} / \mathrm{s}$ and weights of $101.1 \mathrm{~kg}$. Additionally, lowering the impact duration in the NFL by $0.19 \mathrm{~ms}(6.37$ ms) given average weights and velocities set the injury chance to $50 \%$. Using these values, injury sensitivity analysis, seen in Figure 5 could be conducted. It can be concluded that the velocity and impact duration are highly correlated with injury chance and play the most significant roles on the outcome of a collision. A change in the velocity by just 0.007 $\mathrm{m} / \mathrm{s}$ causes a deviation of $1 \%$ down from $50 \%$ injury levels. The same numbers for weight and impact duration are $0.360 \mathrm{~kg}$ and $-0.004 \mathrm{~ms}$, meaning that shorter impact duration increases the chance of injury. Using standard SI units, this shows that the velocity of a player has a 51 times larger impact on concussion risks than weight. Implications of this finding suggest that a player in a helmet to helmet collision with a high velocity, such as during a full sprint or leap, is at an especially high risk for head injury when compared to slower impacts.

While velocity was shown to be the key player variable for brain injury, injury calculation was shown to most sensitive to a collision's impulse duration. This statistic is closely tied to the helmet and its materials. Sub-par or out of date helmets where the padding has worn down would greatly increase the risk of injury. Based on these sensitivity values, it can be concluded that concussion chance can be greatly lowered by discontinuing the use of older, worn out helmets, as well as by incentivizing tackles with lower relative velocities between the players.

\section{Youth players}

The durations of an impact needed to cause injury in children, as seen in Figure 6, are significantly lower than those found in the NFL data due to the much lower weights and velocities. However, the equipment used by youths is often secondhand or otherwise substandard, so the ability of a helmet used by youths to prevent forces from reaching the brain may be lower than that of an NFL helmet. Additionally, because of the substandard equipment, the impact duration over which forces are dissipated may be decreased. Both the increase of transmitted force and the decreased impact duration would result in a more dangerous collision and increased chances of injury development.

As a child grows, the concussion risk was found to increase, both due to an increase in weight and average velocity. Since growth and average weights and velocities vary between the two sexes, this also predicts a slight difference in concussion risk between males and females given identical impacts, with females experiencing a slightly greater risk than males for most stages of development. Because of this discrepancy, testing should be conducted to see if both sexes are adequately protected given a collision.

\section{Conclusion}

The analysis here shows the risk associated with concussion in contact sports with possible head to head collisions. Monte Carlo simulation of $2 \mathrm{~ms}$ duration NFL collisions gave a range of impacts that approximated a normal distribution with a mean of 125 g's and standard deviation of 20 g's. Using the rheological model of force reduction generated here, impacts were translated into injury chances based on the weight, velocity, and duration of impact. Analysis of these factors revealed the values that yield a 50\% injury chance to be $6.37 \mathrm{~ms}$, $8.07 \mathrm{~m} / \mathrm{s}$, and $101.1 \mathrm{~kg}$.
Sensitivity analysis showed the amount by which these values must change to evoke a $1 \%$ injury chance change about these mean values to be $0.360 \mathrm{~kg} / \%$ injury for weight, $0.007(\mathrm{~m} / \mathrm{s}) / \%$ injury for velocity, and $-0.0040 \mathrm{~s} / \%$ injury for time. Thresholds for youths showed a 50\% injury chance to be $3.38 \mathrm{~ms}$ for a 90th percentile weight, 15 year old male and $2.48 \mathrm{~ms}$ for a 10 year old, 50th percentile male. The same inputs for females give values of $3.45 \mathrm{~ms}$ and $3.00 \mathrm{~ms}$ respectively. Overall, females were predicted to be slightly more sensitive to concussion due to average weights and velocities.

\section{References}

1. Guskiewicz KM, Weaver NL, Padua DA, Garrett WE Jr (2000) Epidemiology of concussion in collegiate and high school football players.Am J Sports Med 28: 643-650.

2. Lincoln AE, Caswell SV, Almquist JL, Dunn RE, Norris JB, et al. (2011) Trends in concussion incidence in high school sports: a prospective 11-year study.Am J Sports Med 39: 958-963.

3. Doolan AW, Day DD, Maerlender AC, Goforth M, Gunnar Brolinson P (2012) A review of return to play issues and sports-related concussion.Ann Biomed Eng 40: 106-113.

4. Barth JT, Freeman JR, Broshek DK, Varney RN (2001) AccelerationDeceleration Sport-Related Concussion: The Gravity of It All.J Athl Train 36: 253-256.

5. Prins ML, Hovda DA (2003) Developing experimental models to address traumatic brain injury in children.J Neurotrauma 20: 123-137.

6. Rowson S, Duma SM, Beckwith JG, Chu JJ, Greenwald RM, et al. (2012) Rotational head kinematics in football impacts: an injury risk function for concussion.Ann Biomed Eng 40: 1-13.

7. Broglio SP, Sosnoff JJ, Shin S, He X, Alcaraz C, et al. (2009) Head impacts during high school football: a biomechanical assessment.J Athl Train 44: 342349 .

8. Patel A, Goswami T (2012) Comparison of Intracranial Pressure by Lateral and Frontal Impacts - Validation of Computational Model. Injury and Skeletal Biomechanics. 95-114.

9. National Center for Health Statistics. (2001) Boys Length-for-age and Weightfor-age Clinical Chart. Centers for Disease Control.

10. Moss WC, King MJ (2011) Impact response of US Army and National Football League helmet pad systems. Defense Technical Information Center.

11. Sterken E (2003) From the cradle to the grave: how fast can we run?J Sports Sci 21: 479-491.

12. Coakwell MR, Bloswick DS, Moser R Jr (2004) High-risk head and neck movements at high $\mathrm{G}$ and interventions to reduce associated neck injury.Aviat Space Environ Med 75: 68-80.

13. Marjoux D, Baumgartner D, Deck C, Willinger $R$ (2008) Head injury prediction capability of the HIC, HIP, SIMon and ULP criteria.Accid Anal Prev 40: 11351148.

14. Viano DC, Casson IR, Pellman EJ (2007) Concussion in professional football: biomechanics of the struck player--part 14.Neurosurgery $61: 313-327$. 